\title{
Liver Stiffness Measurement in Psoriasis: Do Metabolic or Disease Factors Play the Important Role?
}

\author{
Jamrus Pongpit, ${ }^{1}$ Saneerat Porntharukchareon, ${ }^{2}$ Piyaporn Kaewduang, \\ Kwannapa Promson, ${ }^{1}$ Wasana Stitchantrakul, ${ }^{3}$ Supanna Petraksa, ${ }^{1}$ \\ Ammarin Thakkinstian, ${ }^{4}$ Chomsri Kositchaiwat, ${ }^{1}$ \\ Natta Rajatanavin, ${ }^{2}$ and Abhasnee Sobhonslidsuk ${ }^{1}$ \\ ${ }^{1}$ Division of Gastroenterology and Hepatology, Department of Medicine, Faculty of Medicine, Ramathibodi Hospital, \\ Mahidol University, Bangkok 10400, Thailand \\ ${ }^{2}$ Division of Dermatology, Department of Medicine, Faculty of Medicine, Ramathibodi Hospital, Mahidol University, \\ Bangkok 10400, Thailand \\ ${ }^{3}$ Research Center, Faculty of Medicine, Ramathibodi Hospital, Mahidol University, Bangkok 10400, Thailand \\ ${ }^{4}$ Section for Clinical Epidemiology and Biostatistics, Faculty of Medicine, Ramathibodi Hospital, Mahidol University, \\ Bangkok 10400, Thailand
}

Correspondence should be addressed to Abhasnee Sobhonslidsuk; abhasnee.sob@mahidol.ac.th

Received 19 September 2015; Accepted 31 January 2016

Academic Editor: Vinod Chandran

Copyright (C) 2016 Jamrus Pongpit et al. This is an open access article distributed under the Creative Commons Attribution License, which permits unrestricted use, distribution, and reproduction in any medium, provided the original work is properly cited.

Background. An increased prevalence of metabolic syndrome including nonalcoholic fatty liver disease (NAFLD) was reported in psoriasis. NAFLD can progress to nonalcoholic steatohepatitis and fibrosis. Transient elastography (TE) is a noninvasive liver fibrosis assessment. We evaluated the prevalence of significant liver fibrosis or high liver stiffness measurement (LSM) using the LSM cutoff over $7 \mathrm{kPa}$ and its associated factors in psoriatic patients. Methods. Subjects underwent TE and ultrasonography. Univariate and multivariate analysis were performed for the associated factors. Results. One hundred and sixty-eight patients were recruited. Three patients were excluded due to TE failure. Mean BMI was $24.8 \pm 4.7 \mathrm{~kg} / \mathrm{m}^{2}$. NAFLD, metabolic syndrome, and diabetes were seen in 105 (63.6\%), $83(50.3 \%)$, and 31 (18.8\%) patients. The total cumulative dose of methotrexate over $1.5 \mathrm{~g}$ was seen in $39(23.6 \%)$ patients. Mean LSM was $5.3 \pm 2.9 \mathrm{kPa}$. High LSM was found in 18 (11.0\%) patients. Waist circumference (OR: 1.24; 95\% CI: 1.11-1.38; $P=0.0002$ ), diabetes (OR: 12.70; 95\% CI: $1.84-87.70 ; P=0.010$ ), and AST (OR: $1.08 ; 95 \%$ CI: $1.02-1.16 ; P=0.017$ ) were associated with high LSM. Conclusion. 11\% of psoriatic patients had significant liver fibrosis by high LSM. Waist circumference, diabetes, and AST level were the independent predictors.

\section{Introduction}

Psoriasis is a chronic inflammatory immune-mediated skin disease affecting $1-3 \%$ of the world population. It is characterized by increases in local and systemic proinflammatory cytokines [1]. The increased levels of proinflammatory cytokines may explain the progression of atherogenesis and the development of peripheral insulin resistance, which can cause hypertension and diabetes $[1,2]$. Recent studies have reported an association between psoriasis and metabolic disorders, such as obesity, dyslipidemia, diabetes, and cardiovascular disorders $[1,3,4]$.

Metabolic syndrome is a risk factor for the development of nonalcoholic fatty liver disease (NAFLD). NAFLD is regarded as the hepatic manifestation of insulin resistance and metabolic syndrome. Metabolic syndrome and NAFLD may coexist in psoriatic patients [5]. Lipotoxicity, oxidative stress, cytokines, and proinflammatory mediators may play major roles in the progression from simple steatosis to nonalcoholic steatohepatitis (NASH), advanced fibrosis, and 
cirrhosis [6]. Psoriasis was reported to be associated with fatty liver and liver fibrosis [7, 8]. Methotrexate is the treatment of choice for moderate-to-severe psoriasis. Unfortunately, liver toxicity is the most important limitation of methotrexate for the treatment of psoriasis [9]. The incidence of methotrexaterelated liver toxicity varies considerably depending on concomitant factors such as heavy alcohol drinking. The recently revised consensus stated that psoriatic patients without concomitant diseases should receive liver biopsy when the total cumulative dose of methotrexate reached 3.5-4 g [10]. Theoretically, psoriatic patients with risk factors for NASH may develop liver fibrosis more rapidly and at a lower cumulative methotrexate dose than those without the risk factors. Patients with high risk conditions should undergo liver biopsy when the total cumulative dose of methotrexate exceeds $1.5 \mathrm{~g}$ [10]. In addition to methotrexate use, metabolic syndrome and psoriasis-related factors, such as the duration and severity of disease, as well as the presence of joint involvement, may play important roles in the development of significant liver fibrosis $[7,11]$.

Liver biopsy is the gold standard for assessing the severity of liver fibrosis [12]. However, the risks of biopsy related major complications $(0.01-0.1 \%)$ limit its use in routine clinical practice [13]. Transient elastography (TE) is a noninvasive tool for the assessment of liver fibrosis that has been evaluated in chronic liver diseases, including psoriasis $[14,15]$. To date, the factors that affect the development of significant hepatic fibrosis in psoriasis patients remain unknown, and the prevalence of significant liver fibrosis in Thai patients with psoriasis has never been studied.

The aims of the present study were to investigate the prevalence of significant liver fibrosis or high liver stiffness measurement (LSM) assessed by TE and to evaluate the factors that were associated with high LSM in patients with psoriasis. The objectives of this study were as follows: (i) to study the liver fibrosis in patients with psoriasis by using the TE; (ii) to compare the characteristics of psoriatic patients with and without liver fibrosis.

\section{Materials and Methods}

2.1. Patients. After informed consent was obtained, psoriatic patients were enrolled consecutively from the psoriasis clinic at Ramathibodi Hospital, which is a tertiary care medical center, between January 1st and December 31st 2013. Inclusion criteria included an age of $\geq 18$ years and a clinical diagnosis of chronic plaque psoriasis. Exclusion criteria included the presence of known liver diseases such as chronic viral hepatitis, having history of heavy alcohol drinking (>20 g of alcohol per day), or having pregnancy. Data including a detailed history of psoriasis, comorbidities, and regular drug use were collected.

2.2. Study Design. A cross-sectional study was conducted in psoriatic patients. The protocol was approved by the hospital ethics committee. The study was carried out according to the Helsinki Declaration (1964) as revised in 2013. Informed consent was obtained from the study participants before enrollment.
2.3. Diagnosis of Psoriasis and the Severity Assessment of the Disease. Psoriasis was diagnosed in accordance with the International Classification of Diseases (the 10th revision). Psoriatic arthritis was evaluated based on the criteria recommended by Moll and Wright [16]. The age of onset, duration of disease, and the total cumulative dose of methotrexate were recorded. The psoriasis area and severity index (PASI) score, a score that assesses erythema, induration, and the scaliness of lesions at 4 body areas (the head, trunk, arms, and legs), was calculated [17]. PASI scores range from 0 to 72 , where a PASI score of $\geq 10$ was defined as severe psoriasis [17].

2.4. Biochemical Testing. Fasting blood tests including alanine aminotransferase (ALT) and aspartate aminotransferase (AST), alkaline phosphatase (ALP), total bilirubin (TB), albumin, gamma-glutamyl transferase (GGT), total, lowdensity lipoprotein (LDL), and high-density lipoprotein (HDL) cholesterol, total triglycerides (TG), and glucose and insulin levels were collected. Insulin resistance was evaluated based on the homeostatic model assessment insulin resistance (HOMA-IR) index using the formula of fasting plasma insulin $(\mathrm{mIU} / \mathrm{L}) \times$ fasting plasma glucose $(\mathrm{mg} / \mathrm{dL}) / 405$ [18]

2.5. Anthropometric Measurement. Height (m), weight (kg), body mass index (BMI) $\left(\mathrm{kg} / \mathrm{m}^{2}\right)$, and waist circumference $(\mathrm{cm})$ were assessed. From the recommendation of the World Health Organization, International Association for the Study of Obesity, and International Obesity Task Force in 2000, the BMI cutoffs of 23.0 to 24.9 and $\geq 25.0 \mathrm{~kg} / \mathrm{m}^{2}$ were defined as overweight and obesity in adult Asians [19]. Metabolic syndrome was diagnosed by the consensus definition incorporating International Diabetes Federation (IDF) and American Heart Association/National Heart, Lung, and Blood Institute (AHA/NHLBI) criteria [20]. The consensus definition consists of three of the following criteria: central obesity (waist circumference $102 \mathrm{~cm}$ in men and 88 women, or $90 \mathrm{~cm}$ in Asian men and $80 \mathrm{~cm}$ in Asian women), TG $150 \mathrm{mg} / \mathrm{dL}$ or on drug treatment for elevated TG, HDL cholesterol $40 \mathrm{mg} / \mathrm{dL}$ in men and $50 \mathrm{mg} / \mathrm{dL}$ in women or on drug treatment for reduced $\mathrm{HDL}$, fasting plasma glucose $100 \mathrm{mg} / \mathrm{dL}$ or on drug treatment for elevated glucose, and systolic $130 \mathrm{mmHg}$ and/or diastolic $85 \mathrm{mmHg}$ or on antihypertensive drug treatment in a patient with a history of hypertension [20].

\subsection{Liver Stiffness Measurement Using Transient Elastography} (TE). LSM were assessed by TE (Fibroscan ${ }^{\circledR}$, Echosen, Paris), which uses an ultrasound transducer that generates vibrations, creating slow elastic shear waves. The median value of LSM was considered to represent the elastic modulus of the liver. TE was performed on the right lobe of the liver through an intercostal space with the patient in the dorsal decubitus position and the right arm maximally abducted. The measured depth of the liver was between 25 and $65 \mathrm{~mm}$. Ten validated measurements were performed. Only the LSM results with 10 validated measurements, a success rate of at least $60 \%$, and an interquartile range (IQR)/median of less than $30 \%$ were considered acceptable [21]. The results were expressed in kilopascals $(\mathrm{kPa})$. TE was evaluated by a single well-trained nurse who was blinded to the patient 
information and had an experience of performing TE in > 1000 cases. The patients underwent TE after at least four hours of fasting [21]. In this study, significant liver fibrosis was defined by high LSM, which used the cutoff of LSM > $7 \mathrm{kPa}$ according to the reports of TE in psoriatic and NAFLD patients $[14,22]$. LSM $>9.5 \mathrm{kPa}$ was taken as a cutoff for the presence of advanced liver fibrosis, and LSM $>13.0 \mathrm{kPa}$ was used for the presence of cirrhosis according to previous studies of TE in chronic liver diseases and NAFLD [23, 24]. Patients who had LSM $>7 \mathrm{kPa}$ were encouraged to have liver biopsy but this was not mandatory.

2.7. Diagnosis of Fatty Liver by Ultrasonography. The diagnosis and grading of fatty liver were performed with ultrasonography (US) based on the degree of brightness or diffusedly increased echogenicity of the liver parenchyma, echogenic discrepancy of the liver and the kidney, and loss echogenicity of portal venous walls [25].

2.8. Statistical Analysis. Sample size calculation was derived from the formula of $N=Z_{\alpha 2}{ }^{2} P Q / \Delta^{2}$, given that $\alpha$ was 0.05 and $Z_{\alpha 2}$ was 1.96. From a report of significant liver fibrosis in psoriatic patients [14], the prevalence $(P)$ was equal to 0.12 , and $Q$ (or $1-P$ ) was equal to 0.88 . As the result, at least 162 psoriatic patients were required to be enrolled in the study. Data were expressed as mean \pm SD, median (range), or $n$ (\%). The means or percentages of baseline data between patients without and with high liver stiffness were compared using Student's $t$-test or Mann-Whitney $U$ test for continuous variables and Chi-square test or Fisher's exact test for categorical variables. Factors with a $P<0.20$ were further selected for multivariate logistic regression analysis. The results are expressed as odds ratios (OR) and 95\% confidence intervals (95\% CI). A $P<0.05$ was considered statistical significance. Statistical analysis was performed using SPSS v.22.0 (SPSS Inc., Chicago, USA).

\section{Results}

3.1. Demographic and Disease Factors. One hundred and sixty-eight patients were enrolled. Three patients were excluded due to TE failure. The mean BMI was $24.8 \pm$ $4.7 \mathrm{~kg} / \mathrm{m}^{2}$ (Table 1). About one-third of the participants (32.1\%) were overweight, and 20 (12\%) patients were obese. The mean waist circumference was $87.0 \pm 12.3 \mathrm{~cm}$. Eightyeight (53.3\%), 55 (33.3\%), and 31 (18.8\%) patients had dyslipidemia, hypertension, and diabetes, respectively. Eighty-three (50.3\%) patients met the criteria of metabolic syndrome [20]. Six $(3.2 \%)$ patients had PASI scores $>10$, which was correlated with disease factors reflecting the severity of psoriasis, for example, hospital admission and the requirement of systemic therapy. Only 39 (23.6\%) patients had taken methotrexate with the total cumulative dose over $1.5 \mathrm{~g}$. The mean LSM was $5.3 \pm 2.9 \mathrm{kPa}$. Eighteen $(10.9 \%)$ patients had significant liver fibrosis defined by high LSM or an LSM $>7 \mathrm{kPa}$. Eleven (6.7\%) patients had LSM > $9.5 \mathrm{kPa}$ and 4 (2.4\%) patients had LSM $>13.0 \mathrm{kPa}$, suggestive of advance liver fibrosis and cirrhosis, respectively. Two patients with LSM of 8.9 and $11 \mathrm{kPa}$ underwent liver biopsy. The pathological reports revealed that Metavir fibrotic staging of F2 and F3 without hepatitis was confirmed.

3.2. Characteristics of Patients without and with High LSM. The characteristics and laboratory results of the psoriatic patients categorized according to the presence of high LSM are shown in Tables 1 and 2. Univariate analysis was performed to explore factors that were associated with high LSM and the results are shown in Tables 1 and 2. Psoriatic patients without high LSM or significant liver fibrosis $(n=147)$ and those with high LSM or significant liver fibrosis $(n=18)$ had similar characteristics in terms of age and sex (Table 1). Almost all of the psoriatic diseaserelated factors (the duration and the severity of psoriasis, the presence of psoriatic arthritis, and the total cumulative dose of methotrexate) except for the PASI score were not different between both groups. From univariate analysis, the factors that were significantly elevated in the high LSM group were BMI, waist circumference, the presence of diabetes, hypertension, dyslipidemia, and metabolic syndrome (Table 1). The PASI score was marginally elevated in the high LSM group $(P=0.042)$. The levels of AST, ALT, fasting glucose, and HOMA-IR and the diagnosis of fatty liver were significantly increased in the high LSM group (Table 2).

3.3. Factors Independently Related to High LSM. Waist circumference, diabetes, AST level, hypertension, dyslipidemia, PASI score, HOMA-IR index, and the diagnosis of fatty liver by US were selected for further analysis with logistic regression model while avoiding the problem of multicollinearity. The multivariate analysis revealed that waist circumference (OR: 1.24; 95\% CI: 1.11-1.38; $P=0.0002$ ), diabetes (OR: 12.70; 95\% CI: $1.84-87.70 ; P=0.010$ ), and AST level (OR: $1.08 ; 95 \%$ CI: $1.02-1.16 ; P=0.017)$ were independently associated with the presence of high LSM or significant liver fibrosis (Table 3).

\section{Discussion}

Previous studies which were carried out in western countries have revealed that central obesity and metabolic syndrome were highly prevalent in psoriatic patients $[2,3,5,7,8$, 26]. Psoriatic patients with central obesity and metabolic syndrome were also at a higher risk of developing liver fibrosis $[16,21,27]$.

For psoriasis patients on long-term methotrexate (MTX), serial liver biopsy helps discontinuation of MTX at the time that the significant liver fibrosis appears [28]. The risk of fibrosis progression in MTX associated hepatotoxicity is very low [28]. The necessity of serial liver biopsy should be reconsidered. It may be more reasonable and practical to do serial measures of the amino-terminal propeptide of type III procollagen (PIIINP) every 3-6 months and perform liver biopsy only when PIIINP increases. So, liver biopsy can be avoided in substantial number of psoriatic patients $[29,30]$.

The study of TE in psoriasis revealed $100 \%$ sensitivity for significant fibrosis but only $67 \%$ specificity [31, 32]. The combining use of TE and Fibrotest is more accurate for monitoring liver fibrosis in psoriatic patients on MTX [15]. Liver biopsy can be done when abnormal results of both 
TABLE 1: Demographic data based on the presence of high liver stiffness measurement (LSM $>7 \mathrm{kPa})$.

\begin{tabular}{|c|c|c|c|c|}
\hline & $\begin{array}{c}\text { All patients } \\
(n=165)\end{array}$ & $\begin{array}{c}\mathrm{LSM} \leq 7 \mathrm{kPa} \\
(n=147)\end{array}$ & $\begin{array}{c}\mathrm{LSM}>7 \mathrm{kPa} \\
(n=18)\end{array}$ & $P$ \\
\hline $\mathrm{Age}$ (years) $^{\dagger}$ & $49.2 \pm 14.0$ & $48.7 \pm 14.1$ & $53.4 \pm 12.7$ & 0.182 \\
\hline Male $^{\ddagger}$ & $75(45.5)$ & $65(44.2)$ & $10(55.6)$ & 0.454 \\
\hline Body mass index $\left(\mathrm{kg} / \mathrm{m}^{2}\right)^{\dagger}$ & $24.8 \pm 4.7$ & $24.0 \pm 4.0$ & $30.77 \pm 5.7$ & $<0.0001$ \\
\hline Waist circumference $(\mathrm{cm})^{\dagger}$ & $87.0 \pm 12.3$ & $85.0 \pm 10.7$ & $104.1 \pm 12.0$ & $<0.0001$ \\
\hline Diabetes mellitus ${ }^{\ddagger}$ & $31(18.8)$ & $22(15)$ & $9(50)$ & 0.001 \\
\hline Hypertension $^{\ddagger}$ & $55(33.3)$ & $43(29.3)$ & $12(66.7)$ & 0.003 \\
\hline Dyslipidemia $^{\ddagger}$ & $88(53.3)$ & $74(50.3)$ & $14(77.8)$ & 0.043 \\
\hline Metabolic syndrome & $83(50.3)$ & $66(44.9)$ & $17(94.4)$ & $<0.0001$ \\
\hline \multicolumn{5}{|l|}{ Psoriasis disease-related factors } \\
\hline Duration of disease (years) $^{\dagger}$ & $16.5 \pm 12.1$ & $16.7 \pm 12.3$ & $14.8 \pm 10.9$ & 0.533 \\
\hline PASI score ${ }^{\dagger}$ & $3.0 \pm 2.7$ & $2.8 \pm 2.6$ & $4.2 \pm 3.2$ & 0.042 \\
\hline Severe psoriasis ${ }^{\ddagger}$ & $6(3.6)$ & $4(2.7)$ & $2(11.1)$ & 0.130 \\
\hline Psoriatic arthritis ${ }^{\ddagger}$ & $35(21.2)$ & $29(19.7)$ & $6(33.3)$ & 0.220 \\
\hline \multicolumn{5}{|l|}{ Cumulative dose of MTX } \\
\hline None & $57(34.5)$ & $51(34.7)$ & $6(33.3)$ & \multirow{3}{*}{0.906} \\
\hline$\leq 1.5 \mathrm{~g}$ & $69(41.8)$ & $62(42.2)$ & $7(38.9)$ & \\
\hline$>1.5 \mathrm{~g}$ & $39(23.6)$ & $34(23.1)$ & $5(27.8)$ & \\
\hline
\end{tabular}

LSM: liver stiffness measurement; PASI: psoriasis area and severity index; and MTX: methotrexate; ${ }^{\dagger}$ mean \pm SD; ${ }^{\ddagger} n(\%)$.

TABLE 2: Laboratory results according to the presence of high liver stiffness measurement (LSM $>7 \mathrm{kPa})$.

\begin{tabular}{|c|c|c|c|c|}
\hline & $\begin{array}{c}\text { All patients } \\
(n=165)\end{array}$ & $\begin{array}{c}\mathrm{LSM} \leq 7 \mathrm{kPa} \\
(n=147)\end{array}$ & $\begin{array}{c}\mathrm{LSM}>7 \mathrm{kPa} \\
(n=18)\end{array}$ & $P$ \\
\hline$\overline{\mathrm{AST}(\mathrm{U} / \mathrm{L})^{\dagger}}$ & $28.2 \pm 13.6$ & $27.1 \pm 10.6$ & $37.6 \pm 26.7$ & 0.002 \\
\hline $\operatorname{ALT}(\mathrm{U} / \mathrm{L})^{\dagger}$ & $41.2 \pm 23.0$ & $39.3 \pm 19.6$ & $56.4 \pm 39.3$ & 0.003 \\
\hline Fasting glucose $(\mathrm{mg} / \mathrm{dL})^{\dagger}$ & $108.4 \pm 38.3$ & $103.8 \pm 29.7$ & $146.0 \pm 69.8$ & $<0.0001$ \\
\hline Triglyceride $(\mathrm{mg} / \mathrm{dL})^{\dagger}$ & $126.1 \pm 67.8$ & $125.5 \pm 68.7$ & $131.3 \pm 61.5$ & 0.712 \\
\hline $\mathrm{TC}(\mathrm{mg} / \mathrm{dL})^{\dagger}$ & $202.8 \pm 42.9$ & $203.6 \pm 42.0$ & $194.0 \pm 49.9$ & 0.431 \\
\hline LDL-C $(\mathrm{mg} / \mathrm{dL})^{\dagger}$ & $126.1 \pm 35.3$ & $126.4 \pm 34.3$ & $123.4 \pm 44.0$ & 0.781 \\
\hline $\mathrm{HDL}-\mathrm{C}(\mathrm{mg} / \mathrm{dL})^{\dagger}$ & $50.5 \pm 13.2$ & $51.1 \pm 13.1$ & $45.4 \pm 13.0$ & 0.095 \\
\hline $\mathrm{HOMA}^{-\mathrm{IR}^{\dagger}}$ & $3.8 \pm 6.1$ & $3.1 \pm 3.4$ & $9.9 \pm 14.8$ & $<0.0001$ \\
\hline $\operatorname{LSM}(\mathrm{kPa})^{\dagger}$ & $5.3 \pm 2.9$ & $4.5 \pm 1.2$ & $11.5 \pm 4.7$ & $<0.0001$ \\
\hline Fatty liver by $\mathrm{US}^{\ddagger}$ & $105(63.6 \%)$ & $89(60.5 \%)$ & $16(88.9 \%)$ & 0.019 \\
\hline
\end{tabular}

LSM: liver stiffness measurement; AST: aspartate aminotransferase; ALT: alanine aminotransferase; TC: total cholesterol; LDL-C: low-density lipoprotein cholesterol; HDL-C: high-density lipoprotein cholesterol; HOMA-IR: homeostatic model assessment insulin resistance; US: ultrasonography; ${ }^{\dagger}$ mean \pm SD; ${ }^{\ddagger} n$ (\%).

TABLE 3: Multivariate logistic regression analysis for the factors associated with high liver stiffness measurement (LSM > $7 \mathrm{kPa})$.

\begin{tabular}{|c|c|c|c|c|c|}
\hline Variable & Coefficient $(\beta)$ & Standard error & $P$ value & Odds ratio & $95 \% \mathrm{CI}$ \\
\hline Waist circumference & 0.21 & 0.06 & 0.0002 & 1.24 & 1.11 to 1.38 \\
\hline Diabetes & 2.54 & 0.99 & 0.010 & 12.70 & 1.84 to 87.70 \\
\hline AST & 0.08 & 0.03 & 0.017 & 1.08 & 1.02 to 1.16 \\
\hline Dyslipidemia & -1.66 & 1.09 & 0.128 & 0.19 & 0.02 to 1.62 \\
\hline Fatty liver by US & -1.64 & 1.23 & 0.185 & 0.19 & 0.02 to 2.18 \\
\hline PASI score & 0.14 & 0.12 & 0.229 & 1.15 & 0.92 to 1.44 \\
\hline HOMA-IR & 0.08 & 0.09 & 0.329 & 1.09 & 0.92 to 1.29 \\
\hline Hypertension & 0.81 & 0.84 & 0.337 & 2.25 & 0.43 to 11.71 \\
\hline
\end{tabular}

LSM: liver stiffness measurement; AST: aspartate aminotransferase; PASI: psoriasis area and severity index; HOMA-IR: homeostatic model assessment insulin resistance; US: ultrasonography. 
tests present [15]. From a survey in dermatologists worldwide, PIIINP and TE were used by only a minority of dermatologists [33]. TE and Fibrotest should be used in routine clinical practice and both methods should be recommended by medical societies.

The prevalence of significant liver fibrosis or high LSM as defined by an LSM $>7 \mathrm{kPa}$ in Thai psoriatic patients was slightly lower than previous studies (10.9\% versus $12-$ $27 \%)[11,14]$. Similarly, the number of advanced liver fibrosis in psoriasis in this study was slightly lower than a recent report (6.7\% versus $8.1 \%)$ [24]. Waist circumference (OR: 1.24; 95\% CI: 1.11-1.38; $P=0.0002$ ) and diabetes (OR: 12.70; 95\% CI: $1.84-87.70 ; P=0.010$ ) were strongly associated with the presence of high liver stiffness or significant liver fibrosis. From this study, we want to highlight that psoriatic patients who had high waist circumference and/or diabetes had the greater risk of developing significant liver fibrosis from TE. Psoriatic patients with diabetes were almost 13 times as likely to have significant liver fibrosis irrespective of other concomitant factors. The influence of diabetes on the progression of liver fibrosis in psoriasis may be explained with the same pathway of insulin resistance and relating cytokines in the pathogenesis of NAFLD and NASH [6]. Noninvasive assessment of liver fibrosis or liver biopsy in psoriatic patients with diabetes should be performed early in their clinical course. The finding of high prevalence of metabolic risk factors (diabetes or central obesity) in psoriatic patients with significant liver fibrosis is consistent with previous reports $[15,29,34]$.

The total cumulative dose of methotrexate and old age were not found to be the risk factors of significant liver fibrosis in this study, which is contrasting with the results of previous studies [24, 27]. From some studies, the total cumulative dose of methotrexate was reported to be an important factor for the presence of liver fibrosis $[10,11,29,34]$. However, there were only 39 (23.6\%) patients with the total cumulative dose of methotrexate over $1.5 \mathrm{~g}$ in this study. It was suggested that methotrexate might accelerate the progression of NASH [11]. This notion leads to a presumption that psoriatic patients who are on methotrexate may be at a greater risk for the progression of liver fibrosis especially those who have concomitant diabetes or metabolic syndrome or are overweight [11,35]. Liver fibrosis in psoriatic patients with $\mathrm{NASH}$ may develop and progress at a lower cumulative dose of methotrexate than those without NASH.

There were some limitations of our study. Firstly, it was a small sample size and single center study. Larger studies are required to confirm the results of our findings. Secondly, TE which is a noninvasive fibrosis assessment tool was used to measure the degree of liver fibrosis in this study. Liver biopsy, a gold standard tool for liver fibrosis assessment, was performed in only 2 of 18 psoriatic patients who had LSM $>7 \mathrm{kPa}$.

\section{Conclusion}

Approximately $11 \%$ of the psoriatic patients had high liver stiffness or significant liver fibrosis. Our study showed that the presence of significant liver fibrosis in patients with psoriasis was strongly associated with waist circumference, diabetes, and AST level. Other psoriasis-related disease factors and the total cumulative dose of methotrexate could not be demonstrated to be related to the development of significant liver fibrosis in psoriatic patients.

$\begin{array}{ll}\text { Abbreviations } \\ \text { NAFLD: } & \text { Nonalcoholic fatty liver disease } \\ \text { NASH: } & \text { Nonalcoholic steatohepatitis } \\ \text { TE: } & \text { Transient elastography } \\ \text { PASI: } & \text { Psoriasis area and severity index } \\ \text { HOMA-IR: } & \text { Homeostatic model assessment insulin } \\ & \text { resistance } \\ \text { BMI: } & \text { Body mass index } \\ \text { LSM: } & \text { Liver stiffness measurement } \\ \text { US: } & \text { Ultrasonography } \\ \text { OR: } & \text { Odds ratio. }\end{array}$

\section{Conflict of Interests}

The authors declare that there is no conflict of interests regarding the publication of this paper.

\section{Authors' Contribution}

All authors contributed substantially to this work and gave approval to the final draft.

\section{Acknowledgments}

The study was funded by the Faculty of Medicine, Ramathibodi Hospital, and the Gastroenterological Association of Thailand.

\section{References}

[1] T. Henseler and E. Christophers, "Disease concomitance in psoriasis," Journal of the American Academy of Dermatology, vol. 32, no. 6, pp. 982-986, 1995.

[2] D. M. Sommer, S. Jenisch, M. Suchan, E. Christophers, and M. Weichenthal, "Increased prevalence of the metabolic syndrome in patients with moderate to severe psoriasis," Archives of Dermatological Research, vol. 298, no. 7, pp. 321-328, 2006.

[3] L. Mallbris, C. T. Ritchlin, and M. Ståhle, "Metabolic disorders in patients with psoriasis and psoriatic arthritis," Current Rheumatology Reports, vol. 8, no. 5, pp. 355-363, 2006.

[4] L. Mallbris, O. Akre, F. Granath et al., "Increased risk for cardiovascular mortality in psoriasis inpatients but not in outpatients," European Journal of Epidemiology, vol. 19, no. 3, pp. 225-230, 2004.

[5] P. Gisondi, G. Tessari, A. Conti et al., "Prevalence of metabolic syndrome in patients with psoriasis: a hospital-based case-control study," The British Journal of Dermatology, vol. 157, no. 1, pp. 68-73, 2007.

[6] G. C. Farrell and C. Z. Larter, "Nonalcoholic fatty liver disease: from steatosis to cirrhosis," Hepatology, vol. 43, no. 2, supplement 1, pp. S99-S112, 2006.

[7] L. Miele, S. Vallone, C. Cefalo et al., "Prevalence, characteristics and severity of non-alcoholic fatty liver disease in patients with chronic plaque psoriasis," Journal of Hepatology, vol. 51, no. 4, pp. 778-786, 2009. 
[8] S. Madanagobalane and S. Anandan, "The increased prevalence of non-alcoholic fatty liver disease in psoriatic patients: a study from South India," Australasian Journal of Dermatology, vol. 53, no. 3, pp. 190-197, 2012.

[9] Q. E. Whiting-O’Keefe, K. H. Fye, and K. D. Sack, "Methotrexate and histologic hepatic abnormalities: a meta-analysis," American Journal of Medicine, vol. 90, no. 1, pp. 711-716, 1991.

[10] R. E. Kalb, B. Strober, G. Weinstein, and M. Lebwohl, "Methotrexate and psoriasis: 2009 National Psoriasis Foundation Consensus Conference," Journal of the American Academy of Dermatology, vol. 60, no. 5, pp. 824-837, 2009.

[11] P. Rosenberg, H. Urwitz, A. Johannesson et al., "Psoriasis patients with diabetes type 2 are at high risk of developing liver fibrosis during methotrexate treatment," Journal of Hepatology, vol. 46, no. 6, pp. 1111-1118, 2007.

[12] E. M. Brunt, C. G. Janney, A. M. Di Bisceglie, B. A. Neuschwander-Tetri, and B. R. Bacon, "Nonalcoholic steatohepatitis: a proposal for grading and staging the histological lesions," The American Journal of Gastroenterology, vol. 94, no. 9, pp. 2467-2474, 1999.

[13] R. J. G. Chalmers, B. Kirby, A. Smith et al., "Replacement of routine liver biopsy by procollagen III aminopeptide for monitoring patients with psoriasis receiving long-term methotrexate: a multicentre audit and health economic analysis," British Journal of Dermatology, vol. 152, no. 3, pp. 444-450, 2005.

[14] M. A. M. Berends, J. Snoek, E. M. G. J. De Jong et al., "Biochemical and biophysical assessment of MTX-induced liver fibrosis in psoriasis patients: fibrotest predicts the presence and Fibroscan ${ }^{\circledR}$ predicts the absence of significant liver fibrosis," Liver International, vol. 27, no. 5, pp. 639-645, 2007.

[15] M. Lynch, E. Higgins, P. A. McCormick et al., "The use of transient elastography and FibroTest for monitoring hepatotoxicity in patients receiving methotrexate for psoriasis," JAMA Dermatology, vol. 150, no. 8, pp. 856-862, 2014.

[16] J. M. H. Moll and V. Wright, "Psoriatic arthritis," Seminars in Arthritis and Rheumatism, vol. 3, no. 1, pp. 55-78, 1973.

[17] T. Fredriksson and U. Pettersson, "Severe psoriasis-oral therapy with a new retinoid," Dermatologica, vol. 157, no. 4, pp. 238$244,1978$.

[18] D. R. Matthews, J. P. Hosker, A. S. Rudenski, B. A. Naylor, D. F. Treacher, and R. C. Turner, "Homeostasis model assessment: insulin resistance and $\beta$-cell function from fasting plasma glucose and insulin concentrations in man," Diabetologia, vol. 28, no. 7, pp. 412-419, 1985.

[19] WHO Expert Consultation, "Appropriate body-mass index for Asian populations and its implications for policy and intervention strategies," The Lancet, vol. 363, no. 9403, pp. 157-163, 2004.

[20] S. M. Grundy, H. B. Brewer Jr., J. I. Cleeman, S. C. Smith Jr., and C. Lenfant, "Definition of metabolic syndrome: report of the National Heart, Lung, and Blood Institute/American Heart Association conference on scientific issues related to definition," Circulation, vol. 109, no. 3, pp. 433-438, 2004.

[21] L. Castéra, J. Vergniol, J. Foucher et al., "Prospective comparison of transient elastography, Fibrotest, APRI, and liver biopsy for the assessment of fibrosis in chronic hepatitis C," Gastroenterology, vol. 128, no. 2, pp. 343-350, 2005.

[22] V. W.-S. Wong, J. Vergniol, G. L.-H. Wong et al., "Diagnosis of fibrosis and cirrhosis using liver stiffness measurement in nonalcoholic fatty liver disease," Hepatology, vol. 51, no. 2, pp. 454-462, 2010.
[23] M. Friedrich-Rust, M.-F. Ong, S. Martens et al., "Performance of transient elastography for the staging of liver fibrosis: a metaanalysis," Gastroenterology, vol. 134, no. 4, pp. 960-974, 2008.

[24] E. A. van der Voort, E. M. Koehler, T. Nijsten et al., "Increased prevalence of advanced liver fibrosis in patients with psoriasis: a cross-sectional analysis from the Rotterdam study," Acta Dermato-Venereologica, vol. 96, no. 2, pp. 213-217, 2015.

[25] L. Needleman, A. B. Kurtz, M. D. Rifkin, H. S. Cooper, M. E. Pasto, and B. B. Goldberg, "Sonography of diffuse benign liver disease: accuracy of pattern recognition and grading," American Journal of Roentgenology, vol. 146, no. 5, pp. 1011-1015, 1986.

[26] A. W. Armstrong, C. T. Harskamp, and E. J. Armstrong, "Psoriasis and metabolic syndrome: a systematic review and metaanalysis of observational studies," Journal of the American Academy of Dermatology, vol. 68, no. 4, pp. 654-662, 2013.

[27] C. M. Maybury, Z. K. Jabbar-Lopez, T. Wong, A. P. Dhillon, J. N. Barker, and C. H. Smith, "Methotrexate and liver fibrosis in people with psoriasis: a systematic review of observational studies," British Journal of Dermatology, vol. 171, no. 1, pp. 17-29, 2014.

[28] M. J. Boffa, R. J. G. Chalmers, N. Y. Haboubi, M. Shomaf, and D. M. Mitchell, "Sequential liver biopsies during longterm methotrexate treatment for psoriasis: a reappraisal," British Journal of Dermatology, vol. 133, no. 5, pp. 774-778, 1995.

[29] H. Zachariae, "Liver biopsies and methotrexate: a time for reconsideration," Journal of the American Academy of Dermatology, vol. 42, no. 3, pp. 531-534, 2000.

[30] P. D. L. Maurice, A. J. Maddox, C. A. Green, F. Tatnall, J. K. Schofield, and D. J. Stott, "Monitoring patients on methotrexate: hepatic fibrosis not seen in patients with normal serum assays of aminoterminal peptide of type III procollagen," British Journal of Dermatology, vol. 152, no. 3, pp. 451-458, 2005.

[31] A. P. J. J. Bray, I. Barnova, R. Przemioslo, and C. T. C. Kennedy, "Liver fibrosis screening for patients with psoriasis taking methotrexate: a cross-sectional study comparing transient elastography and liver biopsy," British Journal of Dermatology, vol. 166, no. 5, pp. 1125-1127, 2012.

[32] B. H. Kaffenberger, J. A. Kaffenberger, H. Wong, W. Jarjour, D. Levin, and M. A. Bechtel, "Magnetic resonance elastography and transient elastography as non-invasive analyses for liver fibrosis: can they obviate the need for liver biopsy in psoriasis patients treated with methotrexate?" International Journal of Dermatology, vol. 54, no. 7, pp. 752-756, 2015.

[33] R. Gyulai, M. Bagot, C. E. M. Griffiths et al., "Current practice of methotrexate use for psoriasis: results of a worldwide survey among dermatologists," Journal of the European Academy of Dermatology and Venereology, vol. 29, no. 2, pp. 224-231, 2015.

[34] M. A. M. Berends, J. Snoek, E. M. G. J. de Jong et al., "Liver injury in long-term methotrexate treatment in psoriasis is relatively infrequent," Alimentary Pharmacology and Therapeutics, vol. 24, no. 5, pp. 805-811, 2006.

[35] G. Langman, P. D. L. M. Hall, and G. Todd, "Role of nonalcoholic steatohepatitis in methotrexate-induced liver injury," Journal of Gastroenterology and Hepatology, vol. 16, no. 12, pp. 1395-1401, 2001. 


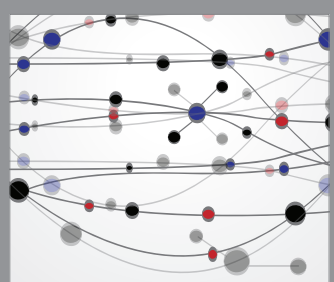

The Scientific World Journal
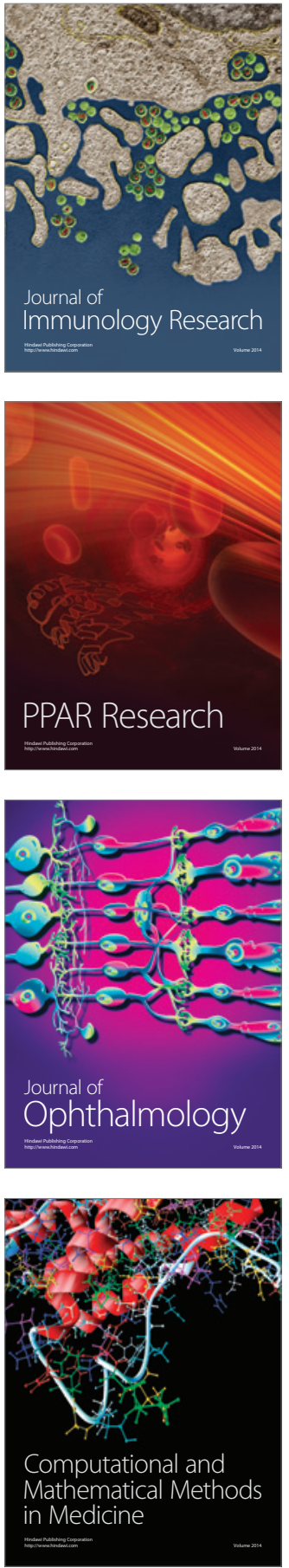

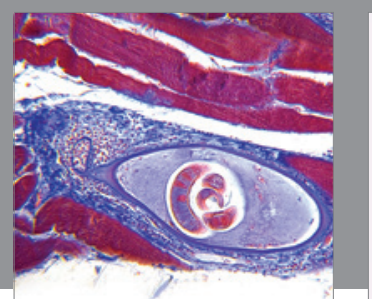

Gastroenterology Research and Practice

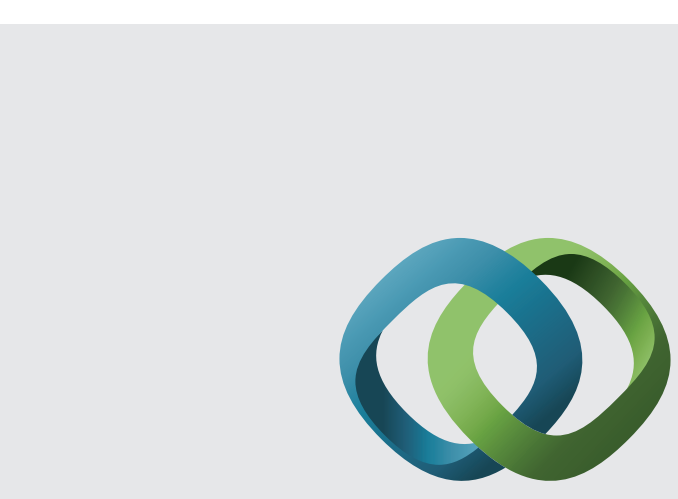

\section{Hindawi}

Submit your manuscripts at

http://www.hindawi.com
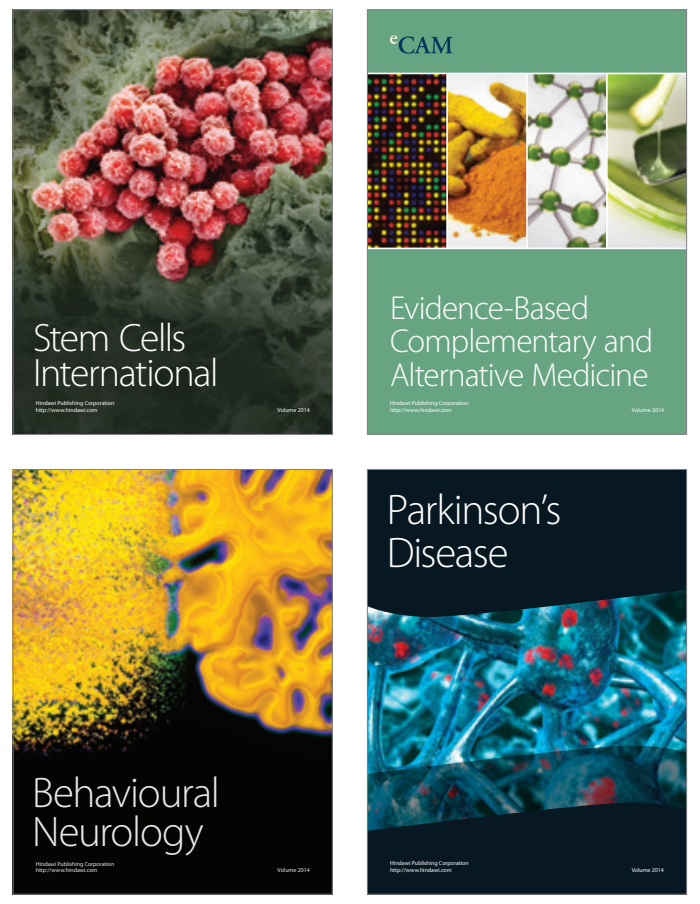
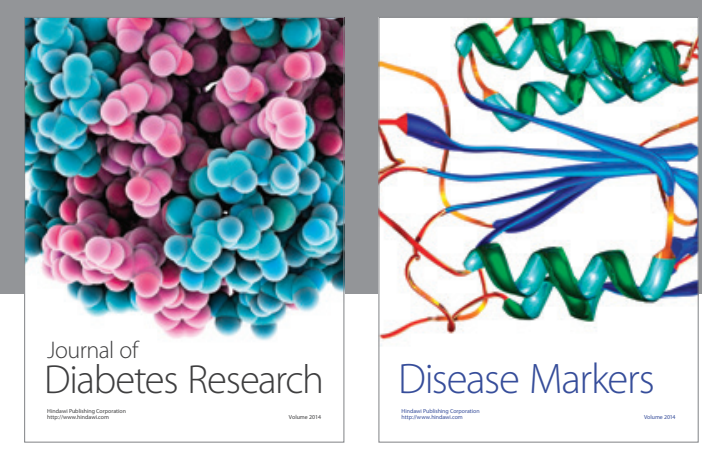

Disease Markers
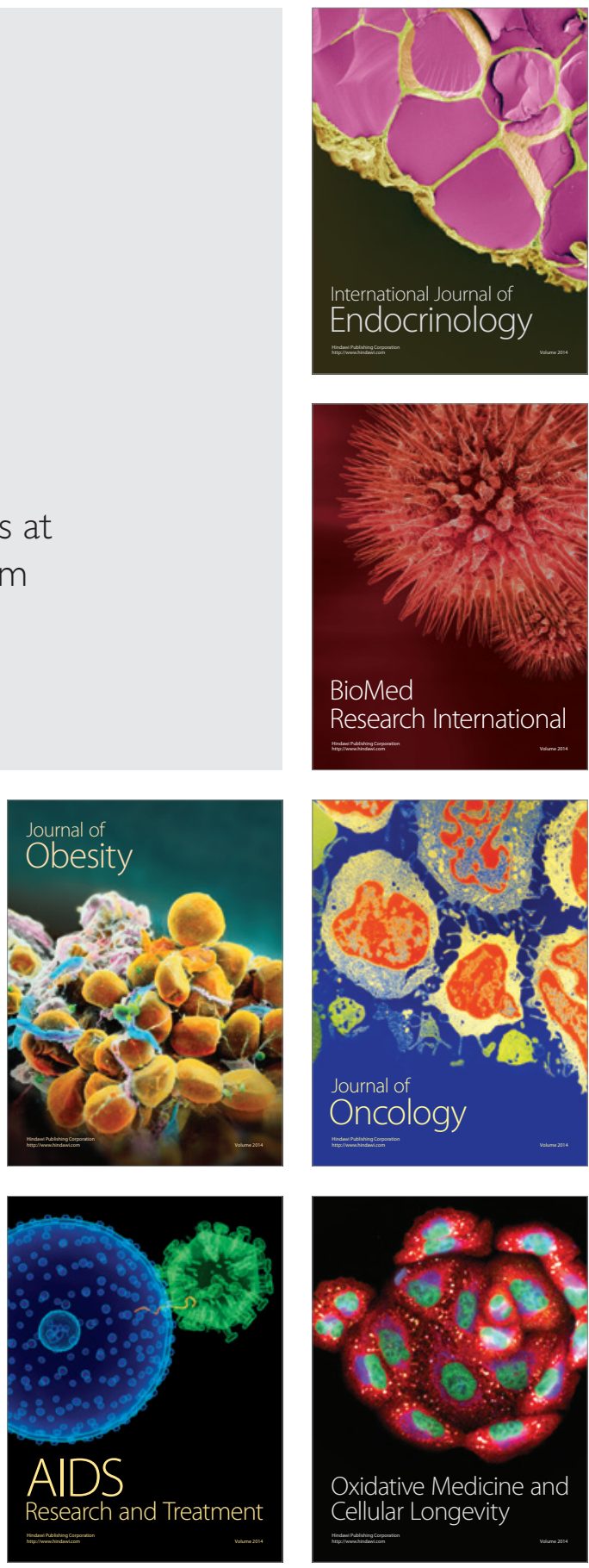